TAMKANG JOURNAL OF MATHEMATICS

Volume 41, Number 4, 375-377, Winter 2010

\title{
FINITE SUMMATION FORMULAE FOR MULTIVARIABLE H-FUNCTION
}

\author{
C. K. SHARMA AND ROSHANI SINGH
}

\begin{abstract}
In the present paper, an attempt has been made to derive finite summation formulae for the $H$-function introduced by H.M. Srivastava and R. Panda [6]. Since the multivariable H- Function includes a large number of a special functions of one and more variables as its particular cases. Therefore, the results established here serve as key formulas giving as a large number of new and interesting results by specializing the parameter involved.
\end{abstract}

\section{Introduction}

For the multivariable $H$-Function, which was introduced by Srivastava and Panda ([6], [7]), is an extension of the Multivariable $G$-function. This multivariable $H$-Function includes Fox's $H$ - and Meijer's $G$-functions of One and Two Variables, the generalized Lauricella function of Srivastava and Daoust [4], Apell function, the Whittaker functions etc. Therefore, the results established in this paper are of general character and hence encompass several cases of interest.

The object of this paper is to establish four finite summation formulae for the multivariable $H$-Function. These formulae will yield a number of new and known results including the results of Gupta and Garg ([2], [3]).

The multivariable $H$-Function define by the Srivastava and Panda ([6], [7]) and represented in the manner already detailed by Srivastava, Gupta and Goyal ([5], p.251). Since only the parameters with subscript 1 in the definition of the of the multivariable $H$-function ([5], p.251) undergo changes in our summation formulae that following, to simplify notational problems, we specify only these parameters in them. Thus $H\left[\left(a_{1}-r ; h, k\right),\left(b_{1}-r ; \beta_{h}, \beta_{k}\right)\right]$ would represent the multivariable $H$-function defined by Srivastava, Gupta and Goyal ([5], p.251) but having $a_{1}$ replaced by $a_{1}-r, \alpha_{1}^{(i)}$ replaced by $h^{(i)}(i=1, \ldots, r), b_{1}$ replaced by $b_{1}-r, \beta_{1}^{(i)}$ replaced by $\beta_{h}^{(i)}(i=1, \ldots, r)$ the rest of the parameters remaining unchanged and so on.

We shall given below three -term contiguous relation for the multivariable $H$-function and use them later on.

(i) $\left[\Gamma\left(1-a_{1}+r+b_{1} / \beta\right)\right]^{-1} H\left[\left(a_{1}+1-r ; h^{(1)}, \ldots, h^{(r)}\right),\left(b_{1}+1 ; \beta_{h^{(1)}}, \ldots, \beta_{h^{(r)}}\right)\right]$

Corresponding author: Roshani Singh.

Received December 12, 2008. 


$$
\begin{aligned}
= & \beta\left[\Gamma\left(1-a_{1}+r+b_{1} / \beta\right)\right]^{-1} H\left[\left(a_{1}-r ; h^{(1)}, \ldots, h^{(r)}\right),\left(b_{1} ; \beta_{h^{(1)}}, \ldots, \beta_{h^{(r)}}\right)\right] \\
& -\beta\left[\Gamma\left(-a_{1}+r+b_{1} / \beta\right)\right]^{-1} H\left[\left(a_{1}-r+1 ; h^{(1)}, \ldots, h^{(r)}\right),\left(b_{1} ; \beta_{h^{(1)}}, \ldots, \beta_{h^{(r)}}\right)\right],
\end{aligned}
$$

(ii) $\beta(-1)^{r}\left[\left(b_{1}+r-a_{1} \beta\right)\right]^{-1} H\left[\left(a_{1}+1 ; h^{(1)}, \ldots, h^{(r)}\right),\left(b_{1}+r-1 ; \beta_{h^{(1)}}, \ldots, \beta_{h^{(r)}}\right)\right]$

$=(-1)^{r}\left[\left(b_{1}+r-a_{1} \beta\right)\right]^{-1} H\left[\left(a_{1}+1 ; h^{(1)}, \ldots, h^{(r)}\right),\left(b_{1}+r ; \beta_{h^{(1)}}, \ldots, \beta_{h^{(r)}}\right)\right]$

$+(-1)^{r}\left[\left(b_{1}+r-1-a_{1} \beta\right)\right]^{-1} H\left[\left(a_{1}+1 ; h^{(1)}, \ldots, h^{(r)}\right),\left(b_{1}+r-1 ; \beta_{h^{(1)}}, \ldots, \beta_{h^{(r)}}\right)\right],(1.2$

(iii) $\left|\begin{array}{cc|}r-b_{1} & 1-a_{1}+r \\ \beta & 1\end{array}\right| H\left[\left(a_{1}-r ; h^{(1)}, \ldots, h^{(r)}\right),\left(b_{1}-r ; \beta_{h^{(1)}}, \ldots, \beta_{h^{(r)}}\right)\right]$

$$
\begin{aligned}
= & H\left[\left(a_{1}-r ; h^{(1)}, \ldots, h^{(r)}\right),\left(b_{1}-r+1 ; \beta_{h^{(1)}}, \ldots, \beta_{h^{(r)}}\right)\right] \\
& -\beta H\left[\left(a_{1}-r-1 ; h^{(1)}, \ldots, h^{(r)}\right),\left(b_{1}-r ; \beta_{h^{(1)}}, \ldots, \beta_{h^{(r)}}\right)\right],
\end{aligned}
$$

(iv) $\quad\left(b_{1}-a_{1}+1\right) H\left[\left(a_{1} ; h^{(1)}, \ldots, h^{(r)}\right),\left(b_{1} ; \beta_{h^{(1)}}, \ldots, \beta_{h^{(r)}}\right)\right]$

$$
=H\left[\left(a_{1}-1 ; h^{(1)}, \ldots, h^{(r)}\right),\left(b_{1} ; \beta_{h^{(1)}}, \ldots, \beta_{h^{(r)}}\right)\right] .
$$

The contiguous relations (1.1), (1.2), (1.3) and (1.4) can be developed on lines similar to those given by Bushman and Gupta [1].

\section{Finite summation formula}

The finite summation formulae to be established are

(i) $\begin{aligned} \sum_{r=1}^{n}\left[\Gamma\left(1-a_{1}+r+b_{1} / \beta\right)\right]^{-1} H\left[\left(a_{1}+1-r ; h^{(1)}, \ldots, h^{(r)}\right),\left(b_{1}+1 ; \beta_{h^{(1)}}, \ldots, \beta_{h^{(r)}}\right)\right] \\ =\beta\left[\left(1-a_{1}+n+b_{1} / \beta\right)\right]^{-1} H\left[\left(a_{1}-n ; h^{(1)}, \ldots, h^{(r)}\right),\left(b_{1} ; \beta_{h^{(1)}}, \ldots, \beta_{h^{(r)}}\right)\right] \\ \quad\left[\left(-a_{1}+b_{1} / \beta\right)\right]^{-1} H\left[\left(a_{1} ; h^{(1)}, \ldots, h^{(r)}\right),\left(b_{1} ; \beta_{h^{(1)}}, \ldots, \beta_{h^{(r)}}\right)\right]\end{aligned}$

(ii) $\beta \sum_{r=1}^{n}(-1)^{r}\left[\left(b_{1}+r-a_{1} \beta\right)\right]^{-1} H\left[\left(a_{1} ; h^{(1)}, \ldots, h^{(r)}\right),\left(b_{1}+r-1 ; \beta_{h^{(1)}}, \ldots, \beta_{h^{(r)}}\right)\right]$

$=(-1)^{n}\left[\left(b_{1}+n-a_{1} \beta\right)\right]^{-1} H\left[\left(a_{1}+1 ; h^{(1)}, \ldots, h^{(r)}\right),\left(b_{1}+n ; \beta_{h^{(1)}}, \ldots, \beta_{h^{(r)}}\right)\right]$ $-\left[\left(b_{1}-a_{1} \beta\right)\right]^{-1} H\left[\left(a_{1}+1 ; h^{(1)}, \ldots, h^{(r)}\right),\left(b_{1} ; \beta_{h^{(1)}}, \ldots, \beta_{h^{(r)}}\right)\right]$

(iii) $\sum_{r=1}^{n}\left|\begin{array}{cc}r-b_{1} & 1-a_{1}+r \\ \beta & 1\end{array}\right| \beta^{r-1} H\left[\left(a_{1}+r ; h^{(1)}, \ldots, h^{(r)}\right),\left(b_{1}-r ; \beta_{h^{(1)}}, \ldots, \beta_{h^{(r)}}\right)\right]$

$=H\left[\left(a_{1}-1 ; h^{(1)}, \ldots, h^{(r)}\right),\left(b_{1} ; \beta_{h^{(1)}}, \ldots, \beta_{h^{(r)}}\right)\right]$

$-\beta n H\left[\left(a_{1}-n-1 ; h^{(1)}, \ldots, h^{(r)}\right),\left(b_{1}-n ; \beta_{h^{(1)}}, \ldots, \beta_{h^{(r)}}\right)\right]$,

(iv) $\sum_{r=1}^{n}(-1)^{r}\left(\begin{array}{l}n \\ r\end{array}\right) H\left[\left(a_{n}-n+r ; h^{(1)}, \ldots, h^{(r)}\right),\left(b_{1}+r ; \beta_{h^{(1)}}, \ldots, \beta_{h^{(r)}}\right)\right]$

$=\left(b_{1}-a_{1}+1\right)_{n} H\left[\left(a_{1}-1 ; h^{(1)}, \ldots, h^{(r)}\right),\left(b_{1} ; \beta_{h^{(1)}}, \ldots, \beta_{h^{(r)}}\right)\right]$. 
Provided that the series involved in all the above formulae is absolutely convergent.

Proof. To prove (2.1), putting $r=1,2, \ldots, n$ in (1.1) in succession and after taking the sum we see that in the resulting series on the right hand side the alternate terms cancel out, and we arrive at the require results (2.1).

Similarly, (2.2) and (2.3) can be established by using the results (1.2) and (1.3) respectively in place of (1.1). [Multiplying by the quantities $1, \beta^{1}, \beta^{2}, \ldots, \beta^{n-1}$ respectively only for (2.3)]. To prove (2.4), if we iterate by expanding term on the right hand side of (1.4) by the use of this varies relation and do not write the repeated parameters

$h_{1}, \ldots, h_{r}$, again and again continuing this process of iteration, we finally arrive at the require result (2.4).

\section{References}

[1] R. G. Buchman and K. C. Gupta, Contiguous relations for the H-function of two variables, Indian J. Pure Appl. Math., 6(1975), 1416-1421.

[2] K. C. Gupta and O. P. Garg, On finite summation formulae for the H-function of two variables, Kyungpook Math. J., 18 (1978), 211-215.

[3] K. C. Gupta and O. P. Garg, On Certain finite series involving the H-function of two variables, Rev. Tech. Fac. Ing. Univ. Zullia, 2(1979), 56-65.

[4] H. M. Srivastava and M. C. Daoust, Certain generalized Neumann expantions associated with the Kampé de Feriet function, Nederi Akad. Wetensch. Proc. Ser. A 72=Indag. Math., 31(1969), 449-457.

[5] H. M. Srivastava, K. C. Gupta and S. P. Goyal, The $H$-functions of One and Two Variables with Applications. South Asian Publishers, New Delhi and Madres, 1982.

[6] H. M. Srivastava and R. Panda, Some bilateral generating functions for a class of generalized hyper geometric polynomials, J. Reine Angew. Math., 283/284(1976), 265-274.

[7] H. M. Srivastava and R. Panda, Some multiple integral transformations involving the $H$ function of several variables, Nederi. Akad. Wetensch. Proc. Ser. A 82=Indag. Math., 41(1979), 353-362.

Department of Mathematics, A. P. S. University, Rewa, (Madhya Pradesh) India.

E-mail: cksharma54@rediffmail.com

Department of Mathematics, A. P. S. University, Rewa, (Madhya Pradesh) India.

E-mail: roshnisinghm@yahoo.com 